\title{
Computational Validation of a 3-D Microwave Imaging System for Breast-Cancer Screening
}

Rubæk, Tonny; Kim, Oleksiy S.; Meincke, Peter

Published in:

I E E E Transactions on Antennas and Propagation

Link to article, DOI:

10.1109/TAP.2009.2021879

Publication date:

2009

Document Version

Publisher's PDF, also known as Version of record

Link back to DTU Orbit

Citation (APA):

Rubæk, T., Kim, O. S., \& Meincke, P. (2009). Computational Validation of a 3-D Microwave Imaging System for Breast-Cancer Screening. I E E E Transactions on Antennas and Propagation, 57(7), 2105-2115.

https://doi.org/10.1109/TAP.2009.2021879

\section{General rights}

Copyright and moral rights for the publications made accessible in the public portal are retained by the authors and/or other copyright owners and it is a condition of accessing publications that users recognise and abide by the legal requirements associated with these rights.

- Users may download and print one copy of any publication from the public portal for the purpose of private study or research.

- You may not further distribute the material or use it for any profit-making activity or commercial gain

- You may freely distribute the URL identifying the publication in the public portal 


\title{
Computational Validation of a 3-D Microwave Imaging System for Breast-Cancer Screening
}

\author{
Tonny Rubæk, Oleksiy S. Kim, and Peter Meincke
}

\begin{abstract}
The microwave imaging system currently being developed at the Technical University of Denmark is described and its performance tested on simulated data. The system uses an iterative Newton-based imaging algorithm for reconstructing the images in conjunction with an efficient method-of-moments solution of the associated forward scattering problem. A cylindrical multistatic antenna setup with 32 horizontally oriented antennas is used for collecting the data. It has been found that formulating the imaging algorithm in terms of the logarithm of the amplitude and the unwrapped phase of the measured signals improves its performance when compared to the more commonly used complex phasor formulation. This improvement is illustrated by imaging a simulated hemispherical breast model using both formulations. In addition to this, the importance of using the correct position and orientation of the antennas in the measurement system is shown by imaging the same breast model using a measurement setup in which the antennas are vertically oriented.
\end{abstract}

Index Terms-Biomedical imaging, cancer, electromagnetic scattering inverse problems, microwave imaging, nonlinear equations.

\section{INTRODUCTION}

B REAST cancer is the most common type of cancer among women [1]-[4], and early detection is one of the most important prerequisites for successful treatment of the cancer [5]. To this end, screening programmes using X-ray mammography is the most widespread approach. $\mathrm{X}$-ray mammography is, however, not perfect, especially when used to screen women under the age of 50 for which sensitivities as low as $53 \%$ have been reported[6]. This leaves room for supplementary imaging modalities, such as the well-established magnetic-resonance and ultrasound imaging techniques[7], albeit these are not suitable for screening.

An emerging imaging modality for breast-cancer screening is microwave imaging which is based on the high contrast in the electromagnetic constitutive parameters between healthy and cancerous breast tissue in the microwave frequency range, reported by several authors [8]-[14]. This contrast causes incident

Manuscript received February 07, 2008; revised October 06, 2008. First published May 12, 2009; current version published July 09, 2009. This work was supported in part by the Danish Technical Research Council, the Trane's Foundation, the Villum Kann Rasmussen Foundation, and in part by the Otto Mønsted Foundation.

T. Rubæk and O. S. Kim are with the Electrical Engineering Department, Technical University of Denmark (DTU) Kgs. Lyngby 2800, Denmark (e-mail: tru@elektro.dtu.dk).

P. Meincke was with the Electrical Engineering Department, Technical University of Denmark (DTU), Kgs. Lyngby 2800, Denmark. He is now with TICRA Engineering Consultants DK-1201 Copenhagen, Denmark.

Color versions of one or more of the figures in this paper are available online at http://ieeexplore.ieee.org.

Digital Object Identifier 10.1109/TAP.2009.2021879 electromagnetic fields in the microwave region of the frequency spectrum to be scattered, allowing for detection of the cancerous tissue. Two different approaches are currently being pursued for microwave imaging of the breast. In one, ultrawideband radar techniques are applied [15]-[23], while in the other, nonlinear inverse scattering techniques, also known as microwave tomography, are used [11], [12], [24], [25].

The inverse scattering techniques seek to determine the distribution of the electromagnetic constitutive parameters, permittivity and conductivity, in the breast. When using this approach, the breast domain is illuminated by one antenna at a time and the response is measured on a number of receiving antennas. These responses may then be used as input to the nonlinear inversion algorithm based on Maxwell's equations. Results for frequency- and time-domain inverse scattering have been reported, both for phantom measurements [25]-[29] and for clinical breast imaging [11], [12], [30] but also for other applications of biomedical imaging [31], [32].

At the Technical University of Denmark (TUD) a microwave imaging system for breast-cancer screening is currently being developed. To reconstruct images of the interior of the breast, frequency-domain nonlinear inverse scattering is applied. The design of the TUD imaging system is inspired by the imaging system currently being used in clinical tests at Dartmouth College. In this system, the breast is imaged using a 2-D measurement setup and reconstructions of the corresponding imaging planes through the breast [11], [28], [30], [33]-[40]. The TUD imaging system, on the other hand, is designed to operate directly on the full 3-D inversion problem, thereby avoiding the inherent errors stemming from applying 2-D algorithms to what is indeed a 3-D problem [41]. The choice of reconstructing the images by solving a full 3-D inverse problem does, however, increase the computational complexity of the problem.

The imaging algorithm is based on a Newton iterative algorithm in which the distribution of the constitutive parameters is updated in each iteration based on a comparison between the measured signals and the signals computed by a forward solver for the current parameter distribution. Since a full 3-D scattering problem must be solved in each iteration of the Newton algorithm, special attention on the computational efficiency of the forward solver is necessary. To this end, a method of moments code, in which the coefficient matrix is reused in each iteration, has been implemented.

During the development of the imaging algorithm it has been found that the widely used complex formulation of the scattering problem, in which complex phasors are used to represent the measured and calculated scattered field, provides suboptimal results. To remedy this, the log-amplitude unwrapped 


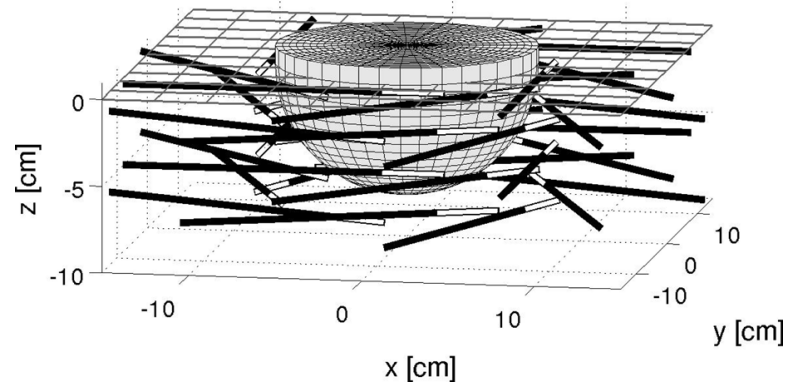

Fig. 1. Schematic of the imaging system. The imaging domain with a radius of $7.5 \mathrm{~cm}$ is indicated by the gray hemisphere and the antennas are given by the black and white lines. The white lines indicate the stripped dielectric and the black indicates the outer conductor. The metallic lid of the measurement tank is indicated by the dark-gray grid at $z=0 \mathrm{~cm}$.

phase, or simply log-phase, formulation, first introduced for 2-D microwave imaging in [42], has been adapted to the three-dimensional imaging algorithm. Although this formulation is just a coordinate transformation, it significantly improves the performance of the reconstruction algorithm.

In this paper, the design of the TUD imaging system is described and the performance of the system is tested on simulated data. This paper is organized as follows: In Section II, the imaging system is presented and in Section III, the Newtonbased imaging algorithm, which may be used with both the complex formulation and the log-phase formulation, is presented. This also includes a presentation of the forward solver as well as the log-phase formulation. Finally, in Section IV, the performance of the system is tested on a simulation of a hemispherical breast model.

\section{IMAGING SYSTEM}

During examination with the TUD microwave imaging system, the patient lies prone atop a measurement tank with her breast suspended through an aperture in the top of the tank. The tank is filled with a glycerin-water coupling liquid to maximize the amount of energy coupled to the interior of the breast. The importance of using such coupling liquid has previously been described by other authors [38], [43]. The system is designed for operation in the frequency domain at a single frequency in the range from $300 \mathrm{MHz}$ to $3 \mathrm{GHz}$ and uses $n_{\text {ant }}=32$ antennas positioned in a cylindrical setup with radius $8 \mathrm{~cm}$. The microwave network of the system is currently under construction but measurements with the prototype indicate that the system will be capable of correctly reconstructing the amplitude and phase of measured signals at levels more than $120 \mathrm{~dB}$ below that of the transmitted signal.

The breast is assumed to be fully contained in a hemispherical imaging domain with a radius of $7.5 \mathrm{~cm}$. A schematic of the imaging system is shown in Fig. 1, with the imaging domain indicated by the gray hemisphere. Although only a single frequency is used for creating the images, the large frequency range allows for choosing the optimum frequency for imaging and for later implementation of multiple-frequency techniques [44], [45], if needed.

The antennas used in the system, one of which is depicted in the photo in Fig. 2, are of a simple monopole type [30]. The an-

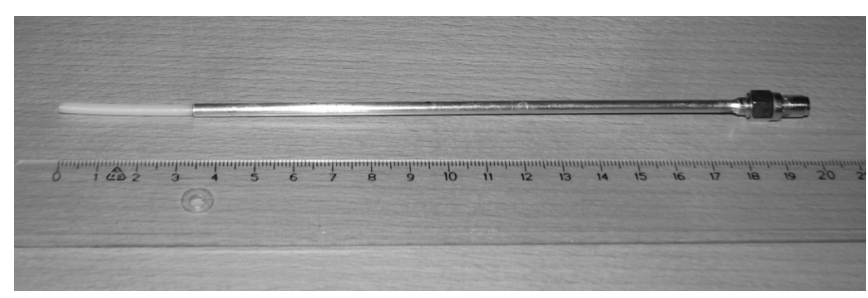

Fig. 2. Photo of one of the antennas of the imaging system. The stripped dielectric has a length of $3.5 \mathrm{~cm}$.

tenna consists of a coaxial cable from which the outer conductor has been stripped from the outermost $3.5 \mathrm{~cm}$ of the antenna, exposing the dielectric. This type of antenna has the advantage of being very simple to manufacture and to include in numerical models. Since the glycerin-water coupling liquid is lossy, the current on the outside of the outer conductor is quickly attenuated and thus, there is no need for a ground plane. Furthermore, when operating in the lossy liquid, the antennas are relatively broadband, allowing for operation in the entire frequency band used by the system. In Fig. 1, the stripped dielectric is represented by the white lines while the outer conductor is represented by the black lines. The 32 antennas are positioned in 4 rows with 8 antennas in each row, with $45^{\circ}$ between the antennas and each row rotated $22.5^{\circ}$ with respect to the row above. The top row of antennas is positioned at $z=-0.5 \mathrm{~cm}$ and the bottom at $z=-7.5 \mathrm{~cm}$ with $z=0 \mathrm{~cm}$ being the surface of the liquid. As seen in Fig. 1, the antennas are $\hat{\boldsymbol{\phi}}$-directed, i.e., horizontally oriented. This is necessary to be able to position the antennas close to the surface of the liquid, thereby obtaining coverage in the entire imaging domain, including the top part. The importance of this is illustrated in Section IV-B.

The antenna system is positioned in a cylindrical measurement tank which has a radius of $20 \mathrm{~cm}$ with each antenna held in place by the coaxial cable. The lossy liquid assures that reflections from the side and bottom of the tank are attenuated so they do not influence the measurements. Therefore, the antenna system may be considered to be positioned in an infinite half space filled with the coupling liquid.

The lid of the measurement tank is made of metal to provide a known boundary toward the chest wall of the patient. Above the $15 \mathrm{~cm}$ diameter aperture, through which the breast is suspended, the imaging domain is extended by a $1 \mathrm{~cm}$ high cylindrical region. It has been found that, due to the shielding effects of the metallic lid, the influence of objects in the upper halfspace outside of this region on the measured signals is minimal. Hence, it is not necessary to include a larger region of the upper halfspace in the reconstruction.

During a measurement, each antenna in turn transmits while the response is measured on the 31 other antennas. This implies that the total measurement consists of $n_{\text {meas }}=992$ measurements of the real and imaginary parts of the received signals.

\section{IMAGING ALGORITHM}

To represent the distribution of constitutive parameters in the imaging domain, the domain is divided into cubic cells. The size of these cells is chosen on the basis of the frequency at 
which the reconstruction is performed and on the expected size of the objects to be imaged. In this paper, the cubic cells are chosen to have a side length of $5 \mathrm{~mm}$, yielding a total of $n_{\text {cells }}=$ 7352 cubic cells in the imaging domain. In each of these cells the constitutive parameters are assumed to be constant and represented by the squared complex wave number of the cell which, when using the time notation $\mathrm{e}^{j \omega t}$, is given by

$$
k^{2}(\boldsymbol{r})=\omega^{2} \mu_{0} \epsilon(\boldsymbol{r})-j \omega \mu_{0} \sigma(\boldsymbol{r}) .
$$

In this expression, $\boldsymbol{r}$ is a position vector, $\omega$ is the angular frequency, $\mu_{0}$ is the free-space permeability, $\epsilon$ is the permittivity, and $\sigma$ is the conductivity. Since the constitutive parameters are assumed to be constant within each cell, it holds that

$$
k^{2}(\boldsymbol{r})=k_{l}^{2}
$$

for

$$
\boldsymbol{r} \in \boldsymbol{R}_{\boldsymbol{l}}
$$

where $\boldsymbol{R}_{\boldsymbol{l}}$ is the volume covered by cell number $l=$ $1,2,3, \ldots, n_{\text {cells. }}$.

The distribution of the squared complex wave numbers is determined by solving the nonlinear minimization problem

$$
\begin{aligned}
\underline{k}^{2}= & \operatorname{argmin}\left\{\left\|\underline{S}^{\text {meas }}-\underline{S}^{\text {calc }}\left(\underline{k}^{2}\right)\right\|_{2}^{2}\right\} \\
= & \operatorname{argmin}\left\{\left\|\underline{S}^{\text {res }}\left(\underline{k}^{2}\right)\right\|_{2}^{2}\right\} \\
& \text { subj. to regularization }
\end{aligned}
$$

using an iterative Newton algorithm. In this expression, the $2 n_{\text {cells }}$ element vector $\underline{k}^{2}$ holds the real and imaginary parts of the squared complex wave numbers, the vector $\underline{S}^{\text {meas }}$ holds the measured signals, while the vector $\underline{S}^{\text {calc }}\left(\underline{k}^{2}\right)$ holds the calculated signals for a given distribution of the squared wave numbers. The vector $\underline{S}^{\text {res }}$ is the residual vector. The nonlinear problem is solved by applying an iterative Newton algorithm, which is initialized with the squared wave numbers in the entire imaging domain set to that of the known coupling liquid, $\underline{k}_{0}$. In each iteration of the Newton algorithm, the distribution of the squared wave numbers is updated using three steps:

1) Compute $\underline{S}^{\text {calc }}\left(\underline{k}_{n}^{2}\right)$ and the Jacobian $\underline{\underline{J}}_{n}$ for the current distribution of squared complex wave numbers.

2) Linearize the problem around the current distribution of constitutive parameters and determine the updates $\Delta k_{n}^{2}$ by solving the linear minimization problem

$$
\underline{\Delta k_{n}^{2}=\operatorname{argmin} \|}\left\|\underline{\underline{J}} \underline{\underline{\Delta}} \underline{k}_{n}^{2}-\underline{S}_{n}^{\mathrm{res}}\right\|_{2}^{2} \text { subj. to regularization. }
$$

This problem is ill-posed and underdetermined with a $2 n_{\text {meas }}$-by- $2 n_{\text {cells }}$ element Jacobian matrix.
3) Update the distribution of the squared wave numbers using

$$
\underline{k}_{n+1}^{2}=\underline{k}_{n}^{2}+\alpha_{n} \underline{\Delta k_{n}^{2}}
$$

wherein $\alpha_{n}$ is the Newton step [46].

Each of the three steps are described in more details below.

\section{A. Log-Phase Formulation}

The complex and log-phase formulations differ in how the data is represented in the vectors $\underline{S}^{\text {meas }}$ and $\underline{S}^{\text {calc }}\left(\underline{k}^{2}\right)$. In the complex formulation, each combination of transmitting antenna $t$ and receiving antenna $r$ yields two elements in each vector, resulting in a total of $2 n_{\text {meas }}$ elements. In $\underline{S}^{\text {meas }}$, the elements are given by the difference between the measured signals with the object to be examined inserted in the imaging system and the measured signals when the system is empty, i.e.,

$$
S_{\Re}^{t, r}=\operatorname{Re}\left\{S_{\text {obj }}^{t, r}-S_{\text {empty }}^{t, r}\right\}
$$

and

$$
S_{\Im}^{t, r}=\operatorname{Im}\left\{S_{\text {obj }}^{t, r}-S_{\text {empty }}^{t, r}\right\} .
$$

In $\underline{S}^{\text {calc }}$, the elements are given in the same way, except here the signals are calculated using the forward solver and not measured. In this way, the Jacobian matrix used when solving the problem in its complex formulation has a size of $2 n_{\text {meas }} \times 2 n_{\text {cells }}$ and consists of the derivative of the real and imaginary parts of the calculated fields with respect to the real and imaginary parts of the squared complex wave numbers.

In the log-phase formulation, each measurement is also represented by two elements in the vectors $\underline{S}^{\text {meas }}$ and $\underline{S}^{\text {calc }}\left(\underline{k}^{2}\right)$, both based on the logarithm of the complex signals. By taking the logarithm of the measured or calculated signals, a complex number is obtained

$$
\log S^{t, r}=\log \left|S^{t, r}\right|+j \angle S^{t, r} \text { empty }
$$

wherein $\left|S^{t, r}\right|$ is the amplitude of the signal and $\angle S^{t, r}$ is the unwrapped phase of the signal. The $2 n_{\text {meas }}$ elements of the vectors $S^{\text {calc }}$ and $S^{\text {meas }}$ may now be determined as

$$
S_{l}^{t, r}=\log \left|S_{\text {obj }}^{t, r}\right|-\log \left|S_{\text {empty }}^{t, r}\right|
$$

and

$$
S_{L}^{t, r}=\angle S_{\text {obj }}^{t, r}-\angle S_{\text {empty }}^{t, r} .
$$

The different performances of the reconstruction algorithm when the two formulations are applied can be explained by observing their two major differences. First, the complex formulation deals with absolute changes in the signal level whereas the log-phase formulation deals with relative changes. This implies that, when using the complex formulation, a given 
change in the signal level is given the same weight in the reconstruction whether the level of the background signal $S_{\text {empty }}^{t, r}$ is large or small. When using the log-phase formulation, on the other hand, a given change in the signal level is given more weight if the background signal level is low (and the change relatively large) than if the background signal is large (and the change relatively small). The signals in which the scattered field has the largest influence, that is, the signals where the relative change is large, also hold more information about the scattering object [47]. Hence, the log-phase formulation improves the performance of the algorithm by weighting the measurements with a high information level more than those with a low information level.

The other major difference between the two formulations is the way in which they handle phase changes. Since the unwrapped phase is used in the log-phase formulation, it is capable of reconstructing images even when the phase in the measured signals changes more than $\pm \pi$, i.e., by using multiple Riemann sheets in the reconstruction. In the complex formulation, on the other hand, the phase information is not retained and all signals will therefore be mapped onto the same Riemann sheet. This implies that if a phase change of more than $\pm \pi$ is encountered, the algorithm will either not reach convergence or reach an erroneous solution since the solution is constrained to a single Riemann sheet. In terms of computational demands, the two formulations are very similar. The log-phase formulation requires that the Jacobian is expressed in terms of the derivatives of the logarithm of the amplitude and phase with respect to the squared complex wave numbers. This can be done using the chain rule, yielding

$$
\frac{\mathrm{d}(\log |S|)}{\mathrm{d}\left(k^{2}\right)}=\frac{1}{|S|^{2}} \operatorname{Re}\left\{S^{*} \frac{\mathrm{d} S}{\mathrm{~d}\left(k^{2}\right)}\right\}
$$

and

$$
\frac{\mathrm{d}(\angle S)}{\mathrm{d}\left(k^{2}\right)}=\frac{1}{|S|^{2}} \operatorname{Im}\left\{S^{*} \frac{\mathrm{d} S}{\mathrm{~d}\left(k^{2}\right)}\right\}
$$

wherein $S^{*}$ denotes the complex conjugate of $S$ and $\mathrm{d} S / \mathrm{d}\left(k^{2}\right)$ is found using (11).

\section{B. Phase Unwrapping}

The phase unwrapping is the one of the most challenging aspects of using the log-phase formulation. Three different methods are applied when unwrapping the phase: Unwrapping in space, unwrapping in frequency, and unwrapping in contrast. When the phase is unwrapped in space, it is assumed that the difference in phase of the signals measured by adjacent antennas is no more than $\pm \pi$. The spatial unwrapping approach is used for unwrapping the measured signals, especially at the lower frequencies, and can easily be implemented in a preprocessor.

When operating at the higher frequencies, the phase of the measured signals may change more than $\pm \pi$ between two adjacent antennas. In this case the phase is unwrapped in frequency instead of in space. This implies that the phase is first unwrapped in space at a low frequency where the phase change between the antennas is within the $\pm \pi$ range. The frequency is then increased in small steps, e.g., $200 \mathrm{MHz}$, and the signals measured by the individual antennas are assumed to change no more than $\pm \pi$ in each frequency step. Although the constitutive parameters of both the background and the breast are frequency dependent, unwrapping in frequency is a useful tool as long as the frequency steps are kept small.

During the image reconstruction, the distribution of squared complex wave numbers is changed in steps, with each iteration of the Newton algorithm changing the distribution of the wave numbers in the imaging domain. This allows for the phase to be unwrapped in contrast in the following manner: Assuming that the phase on any given transmit-receive combination of antennas changes no more than $\pm \pi$ between two successive Newton iteration, the phase of the calculated signals may be unwrapped by comparing the new phase with the phase of the signal in the previous Newton iteration.

When the phase is unwrapped in terms of the contrast it is important that the phase does not change more than $\pm \pi$ between two Newton iterations, and to this end, the scaling of the calculated update with the Newton step, described below, plays an important role.

\section{Forward Solver}

To calculate the forward solution $\underline{S}^{\text {calc }}$, Maxwell's equations are formulated as volume integral equations (VIE) and solved using a method of moments (MoM) code. Other techniques have also been applied for solving the forward problem in inverse scattering, such as the finite-difference time domain method [26], the finite-difference frequency domain method [48], and the hybrid element method [49]. The choice of using a MoM code in the TUD system is to a large extent based on the fact that the department has build up a considerable expertise within this field during the last 10 years [50]-[52].

Solving the forward scattering problem using VIE and MoM consists of two time consuming tasks; filling the MoM matrix and solving the resulting matrix equation

$$
\underline{\underline{Z}} \underline{D}=\underline{E}^{i} .
$$

In this expression, $\underline{Z}$ is the MoM coefficient matrix, $\underline{E}^{i}$ is a vector holding the known incident field, and $\underline{D}$ is a vector holding the unknown dielectric flux density. The most time-consuming task when using MoM for solving the forward problem is the filling of the coefficient matrix. To minimize the impact of this operation on the overall performance of the algorithm, a scheme has been implemented in which the coefficient matrix is calculated only once for a given antenna setup and background medium and then reused in subsequent calls to the forward solver.

In addition to the reuse of the MoM coefficient matrix, the use of higher-order basis functions and the adaptive integral method improves the performance of the algorithm [52]. The benefits of the higher-order basis functions are more pronounced for modeling the relatively large lid of the measurement tank and the long straight antennas while the adaptive integral method assures that the cell size of the imaging domain can be made small 
without causing a prohibitively large, i.e., memory consuming, coefficient matrix.

In a typical setup, filling the coefficient matrix consumes approximately 2 hours while the solution to the forward problem, including calculation of the Jacobian, is obtained in approximately 15 minutes in the subsequent calls to the MoM solver.

When the forward solution has been found in terms of the S-parameters of the 992 combinations of transmitting and receiving antennas, the elements of the Jacobian matrix are found using the expression derived in [53], i.e.,

$$
\frac{\mathrm{d} S^{t, r}}{\mathrm{~d} k_{l}^{2}}=\frac{Z_{c}\left\langle\boldsymbol{E}^{\boldsymbol{t}}, \boldsymbol{E}^{\boldsymbol{r}}\right\rangle_{l}}{2 j \omega \mu_{0} V_{t} V_{r}}
$$

In this expression, the inner product is taken over the cell $l$ and $\boldsymbol{E}^{\boldsymbol{t}}$ and $\boldsymbol{E}^{\boldsymbol{r}}$ are the calculated fields in the cell when the antennas $t$ and $r$ are transmitting. The quantities $V_{t}$ and $V_{r}$ are the voltages of the wave incident on the antennas through the coaxial cable, which has characteristic impedance $Z_{c}$, when calculating the fields $\boldsymbol{E}^{\boldsymbol{t}}$ and $\boldsymbol{E}^{\boldsymbol{r}}$, respectively. The inner product is calculated using the flux density $\boldsymbol{D}$ found when solving the forward problem and the known constitutive parameters of the individual cells.

\section{Calculation of Updates}

To determine the updates, the under-determined and ill-posed problem (4) is solved using the two-step algorithm described in [54]. This algorithm is based on the conjugated gradient least squares (CGLS) algorithm which is an iterative algorithm that solves the linear inversion problem in five simple matrix-vector operations in each CGLS iteration [55, Sec. 6.3]. The solution of the linear inversion problem (4) obtained after $m$ CGLS iterations is given by

$$
\left[\underline{\Delta k}_{n}^{2}\right]^{(m)}=\operatorname{argmin}\left\|\underline{\underline{J}} \underline{\Delta k_{n}^{2}}-\underline{S}^{\mathrm{res}}\left(\underline{k}_{n}^{2}\right)\right\|_{2}
$$

subject to

$$
\left[\underline{\Delta k}_{n}^{2}\right]^{(m)} \in \mathfrak{K}_{m}\left\{\underline{\underline{J}}^{T} \underline{\underline{J}}, \underline{\underline{J}}^{T} \underline{S}^{\mathrm{res}}\left(\underline{k}_{n}^{2}\right)\right\} .
$$

Hence, the solution obtained is the least squares solution of the linear inversion problem with the constraint that the solution must lie in the $m$-dimensional Krylov subspace $\mathfrak{K}_{m}$ defined by the Jacobian matrix and the residual vector. The regularizing effect of the CGLS algorithm is obtained because the $m$-dimensional Krylov subspace can be seen as an approximation of the subspace spanned by the first $m$ right-hand singular vectors. Thus, the solution obtained using the CGLS algorithm can be regarded an approximation of the solution obtained using the truncated singular value decomposition with the important difference that it is not necessary to perform a time consuming singular value decomposition of the problem [55]. As the number of iterations with the CGLS algorithm increases, the amount of regularization applied to the problem decreases.

In [54], the algorithm was applied for 2-D microwave imaging but it has been found that the two-step approach is also valid when the algorithm is used for 3-D imaging: In the first few Newton iterations, the number of CGLS iterations used to calculate the updates is kept low. After a number of Newton iterations, the change in the norm of the residual vector between successive Newton iterations will become small. This indicates that the reconstruction algorithm has reconstructed the major features of the image and the number of CGLS iterations used to calculate the updates is now increased to allow the algorithm to reconstruct the finer details of the image.

In the three-dimensional reconstruction algorithm, it has been found that using 2 CGLS iterations in the first part of the reconstruction and 8 in the latter part yields the optimum results. As it was shown in [54], the change between the low and high number of CGLS iterations is most effectively chosen based on the relative change in the normalized norm of the residual vector. The normalized norm $\eta_{n}$ is introduced as

$$
\eta_{n}=\frac{\left\|\underline{S}^{\mathrm{res}}\left(\underline{k}_{n}^{2}\right)\right\|_{2}}{\left\|\underline{S}^{\mathrm{res}}\left(\underline{k}_{0}^{2}\right)\right\|_{2}}
$$

wherein $\underline{S}^{\text {res }}\left(\underline{k}_{0}^{2}\right)$ is the residual vector for the system simulated with no object inserted. The change between successive Newton iterations, $\Delta \eta_{n}$, may now be introduced as

$$
\Delta \eta_{n}=\frac{\eta_{n}-\eta_{n-1}}{\eta_{n-1}}
$$

As the reconstruction algorithm progresses, the norm of the residual vector decreases, implying a negative value of $\Delta \eta_{n}$. When the value of $\Delta \eta_{n}$ obtains a value greater than -0.1 , the number of CGLS iterations used to calculate the updates is changed from 2 to 8 .

The values of $\eta_{n}$ and $\Delta \eta_{n}$ are also used to determine when to terminate the algorithm: When $\eta_{n}$ drops below 0.02 or if $\Delta \eta_{n}$ becomes greater than -0.03 after the change from 2 to 8 CGLS iterations has been made, the algorithm is terminated. These threshold values are dependent on the noise level in the system and the accuracy of the forward model: If the noise level is decreased or the accuracy of the forward model improved, these value may be set closer to 0 , and vice versa.

\section{E. Newton Step}

The scaling of the update $\Delta k_{n}^{2}$ with the Newton step $\alpha_{n}$ in (5) serves two purposes. First, it serves to assure that the algorithm does not overshoot the actual solution and start to diverge. Second, the Newton step is used to assure that the phases of the calculated signals between two successive Newton iterations do not change too much, thereby ruining the possibility of unwrapping the phase in contrast.

It has been found that the best way to determine the size of the Newton step is by assuming a linearized phase change and calculating the maximum phase change caused by the update $\Delta k_{n}^{2}$. To estimate the maximum phase change caused by the update vector, a new $n_{\text {meas }} \times 2 n_{\text {cells }}$ element matrix $\underline{\underline{J}}_{n}^{L}$ is created from the Jacobian by retaining only those rows that relate to the phase of the calculated signals. The assumed maximum phase change is then determined by multiplying $\underline{\underline{J}}_{n}^{L}$ with the update 
vector and finding the maximum absolute value in the resulting vector

$$
\angle_{\max }=\max \left\{\left|\underline{\underline{J}}_{n}^{L} \underline{\underline{\Delta}}_{n}^{2}\right|\right\} .
$$

If $L_{\max }$ exceeds a given value, e.g., 1 (radian), the Newton step is applied to assure that the phase change is kept at an acceptable level while a value of $L_{\max }$ less than the threshold results in a unity Newton step, i.e.,

$$
\alpha_{n}=\left\{\begin{array}{ll}
1 & \text { for } L_{\max } \leq 1 \\
\frac{1}{L_{\max }} & \text { for } L_{\max }>1
\end{array} .\right.
$$

When the complex formulation is used in the imaging algorithm, the change in phase between two successive Newton iterations is no longer important. In this case, the main purpose of scaling the update by the Newton step is to avoid the reconstruction algorithm getting stuck in a local minimum or that an oscillating, diverging solution is obtained [46, Ch. 1-2], [56, Ch. 5-6], [57]. This implies that the Newton step may be determined by simply observing the ratio between the norm of the update vector $\underline{\Delta k_{n}^{2}}$ and the norm of the parameter vector $\underline{k}_{n}^{2}$. If this ratio exceeds 0.5 the Newton step is used to scale the update, otherwise the Newton step is kept at unity

$$
\alpha_{n}=\left\{\begin{array}{ll}
1 & \text { for } \frac{\left\|\underline{\Delta} k_{n}^{2}\right\|_{2}}{\left\|\underline{k}_{n}^{2}\right\|_{2}} \leq 0.5 \\
\frac{\left\|\underline{k}_{n}^{2}\right\|_{2}}{2 \| \underline{\Delta k_{n}^{2} \|_{2}}} & \text { for } \frac{\left\|\underline{\Delta} k_{n}^{2}\right\|_{2}}{\left\|\underline{k}_{n}^{2}\right\|_{2}}>0.5
\end{array} .\right.
$$

The importance of using the Newton step is reduced by the fact that an over-regularized solution to the linear update problem (4) is found because only 2 iterations are used in the CGLS algorithm. This reduces the chances of the reconstruction algorithm getting stuck in a local minimum, as it was shown in [54].

\section{IMAGING RESULTS}

Since the microwave circuitry of the imaging system is under construction, the performance of the system will be tested using simulated data. The difference between using the log-phase and the complex formulation of the scattering problem is demonstrated using a simulation of the hemispherical breast model shown in Fig. 3. In this simulation, the breast was modeled as a heterogeneously dense breast with a tumor of radius $1 \mathrm{~cm}$ and a fibroglandular inclusion of radius $2 \mathrm{~cm}$ positioned inside. The breast was centered in the imaging system and the tumor and fibroglandular inclusion were centered at $(x, y, z)=$ $(3 \mathrm{~cm}, 0 \mathrm{~cm},-1 \mathrm{~cm})$ and at $(x, y, z)=(-2 \mathrm{~cm}, 0 \mathrm{~cm},-3 \mathrm{~cm})$, respectively. The simulation was done at $1.3 \mathrm{GHz}$, using the constitutive parameters for the average breast and fibroglandular tissue given in [11]. The breast was modeled with a mean value of the relative permittivity of 12.5 and a mean conductivity of $0.35 \mathrm{~S} / \mathrm{m}$. The corresponding values for the fibroglandular tissue were 21.0 and $0.6 \mathrm{~S} / \mathrm{m}$. The constitutive parameters of the individual cells representing the breast tissue and the fibroglandular tissue were assigned using a normal distribution with a standard deviation of $10 \%$ of the mean values to represent the natural

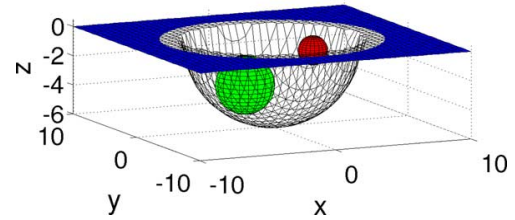

Fig. 3. Schematic of the hemispherical breast model. Inside of the $6 \mathrm{~cm}$ radius breast is a $2 \mathrm{~cm}$ radius fibroglandular inclusion (given by the green sphere) and a $1 \mathrm{~cm}$ radius tumor (given by the red sphere). The breast is centered in the 15 $\mathrm{cm}$ diameter aperture of the metallic lid of the imaging system which is given by the blue surface. To simulate the presence of the chest wall, the relative permittivity and conductivity of the upper half space $(z>0)$ is set to 50 and $5 \mathrm{~S} / \mathrm{m}$, respectively.

variations in the tissue. The tumor was modeled with a relative permittivity of 56.0 and a conductivity of $3.0 \mathrm{~S} / \mathrm{m}$. The coupling liquid was assigned a relative permittivity of 19.6 and a conductivity of 1.2 , corresponding to a $85-15$ glycerin-water mixture. The presence of the chest wall was simulated by assigning the upper halfspace a relative permittivity of 50 and a conductivity of $5 \mathrm{~S} / \mathrm{m}$ while the outermost $3 \mathrm{~mm}$ of the hemispherical breast was assigned a relative permittivity of 30 and a conductivity of $0.8 \mathrm{~S} / \mathrm{m}$ to simulate the skin.

To avoid the "inverse crime", the meshes used to model the breast, lid, and antennas in the simulation were different and finer than those used in the reconstructions. A total of 8908 cells were used to model the $5 \mathrm{~cm}$ radius breast, including the tumor and the fibroglandular inclusion. The antennas were modeled in full in both the simulations and in the reconstructions. The outer conductor was modelled as a thin metal cylinder (radius $1.5 \mathrm{~mm}$ ) and the $3.5 \mathrm{~cm}$ stripped inner conductor modelled as a thinner metal cylinder (radius $0.46 \mathrm{~mm}$ ) surrounded by a cylinder of Teflon with the same radius as the outer conductor and a relative permittivity of 2 and zero conductivity. The length of the cylinder modelling the outer conductor was $12 \mathrm{~cm}$ in the simulation while it was $8 \mathrm{~cm}$ in the reconstructions.

Finally, Gaussian noise mimicking a noise floor $110 \mathrm{~dB}$ below the transmitted power has been added to the simulated signals.

\section{A. Log-Phase Versus Complex Formulation}

The reconstructed images of the heterogeneously dense breast model obtained using the log-phase formulation are shown in Fig. 4 and the images obtained using the complex formulation are shown in Fig. 5.

When the log-phase formulation is used, both the breast, the tumor, and the fibroglandular inclusion are easily identified in the resulting images. The effects of the added noise are, however, clearly visible, especially in the permittivity images where the contours of all three objects are somewhat irregular and artifacts are visible both inside and outside of the breast.

The reconstructed size of the tumor is close to the actual size albeit the reconstructed position is shifted in the negative $z$ direction compared to the actual position. The reconstructed permittivity and conductivity of the tumor are considerably lower than the actual values but the fact that a considerable rise in the conductivity is detected implies that the tumor may be distinguished from the fibroglandular inclusion which is seen to give rise to only a minor increase in conductivity. The log-phase 


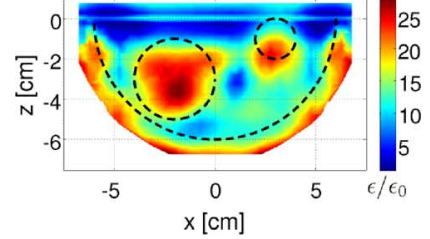

(a) Perm., $y=0 \mathrm{~cm}$

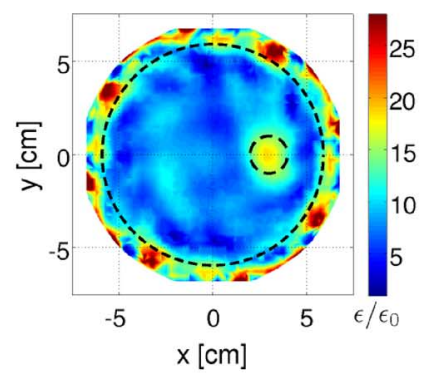

(c) Perm., $z=-1 \mathrm{~cm}$

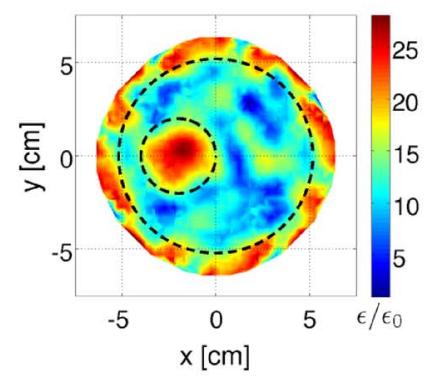

(e) Perm., $z=-3 \mathrm{~cm}$

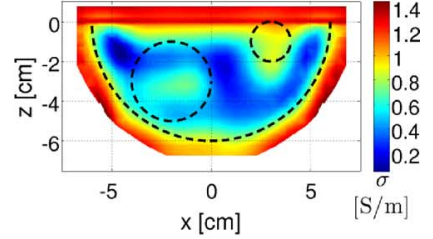

(b) Cond., $y=0 \mathrm{~cm}$

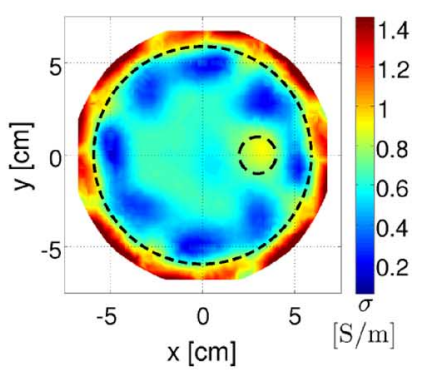

(d) Cond., $z=-1 \mathrm{~cm}$

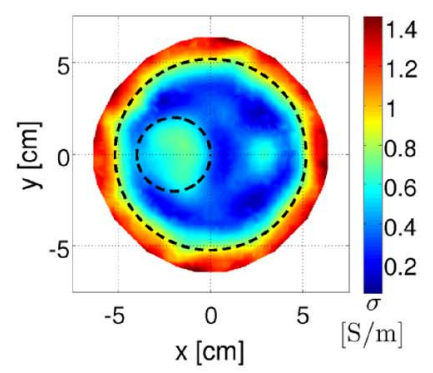

(f) Cond., $z=-3 \mathrm{~cm}$

Fig. 4. Images of the simulated heterogeneously dense breast obtained using the log-phase formulation. The dashed lines indicate the contours of the breast, tumor, and fibroglandular inclusion. Both the tumor and the fibroglandular inclusion are visible, although the reconstructed values of the tumor are somewhat lower than the actual values.

algorithm changes from 2 to 8 iterations in the CGLS algorithm after 7 Newton iterations and reaches convergence after 10 Newton iterations.

In the images reconstructed using the complex formulation, both the breast, fibroglandular inclusion and tumor are reconstructed poorly. This is mainly due to the large phase changes observed in the simulated signals. For neighboring antennas the phase changes are relatively low, but for signals measured with receivers on the opposite side of the breast from the transmitter phase changes of more than $1.5 \pi$ are encountered. This causes problems since the reconstruction is limited to a single Riemann sheet when the complex formulation is applied. The greatest phase changes are obtained when an antenna from the top row is used either as transmitter or receiver, and as a result of this, the top of the breast, including the tumor, is not properly reconstructed. The reconstruction of the bottom of the breast is more successful and an increase in the permittivity at the position of the fibroglandular inclusion is observed, although the circular shape of the inclusion is not reconstructed correctly.

When using the complex formulation, the algorithm changes from the low to the high number of CGLS iterations in the update algorithm after Newton iteration number 13 . The algorithm converges after 19 iterations, and thus uses considerably more time to converge than when the log-phase formulation is applied.

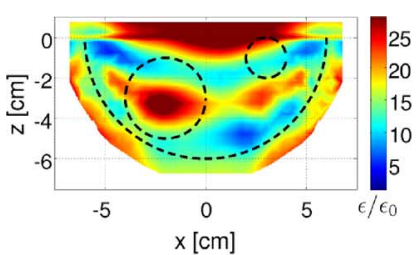

(a) Perm., $y=0 \mathrm{~cm}$

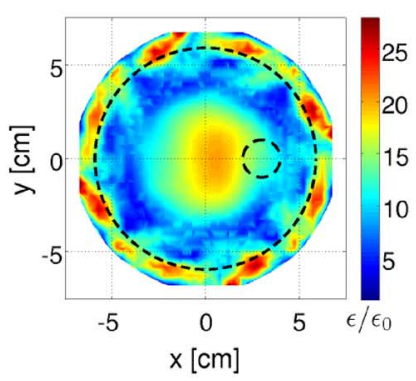

(c) Perm., $z=-1 \mathrm{~cm}$

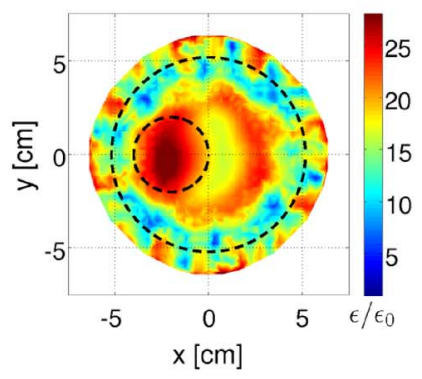

(e) Perm., $z=-3 \mathrm{~cm}$

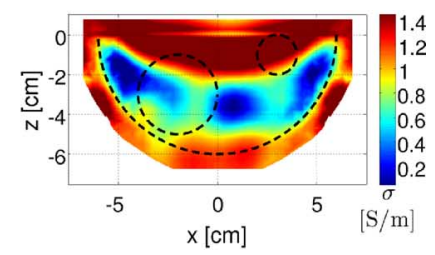

(b) Cond., $y=0 \mathrm{~cm}$

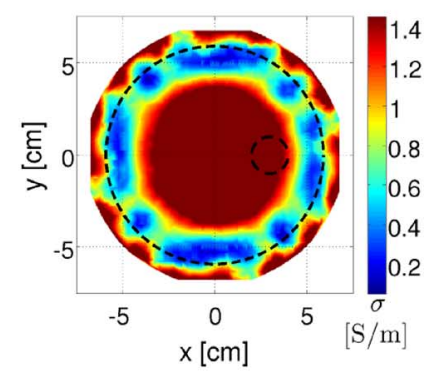

(d) Cond., $z=-1 \mathrm{~cm}$

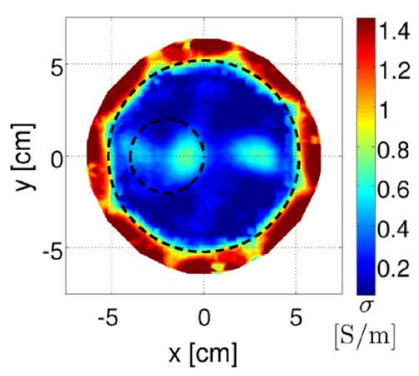

(f) Cond., $z=-3 \mathrm{~cm}$
Fig. 5. Images obtained using the complex formulation. The dashed lines indicate the contours of the breast, tumor, and fibroglandular inclusion. Due to the large phase changes of the measured signals, the algorithm fails to accurately recover the position and size of the tumor and fibroglandular inclusion.

The skin is not visible in either set of images. This is because the reconstructed distributions are low-pass representations of the actual distribution. This implies that the contrast between the high-valued coupling liquid on one side of the skin and the low-valued breast tissue on the other side of the skin results in images where the skin is not visible.

\section{B. Influence of Antenna Configuration}

To illustrate the importance of choosing the optimum antenna configuration, the performance of an antenna configuration with 4 rows of 8 vertically oriented antennas, shown in Fig. 6, has been tested. The antennas were organized in a cylindrical setup with a radius of $8 \mathrm{~cm}$ with the top row of antennas positioned with the tips of the antennas at $z=-1 \mathrm{~mm}$, the second row with the tips of the antennas at $z=-19 \mathrm{~mm}$, the third row with the tips of the antennas at $z=-37 \mathrm{~mm}$, and the bottom row positioned with the tips of the antennas at $z=-55 \mathrm{~mm}$.

The images obtained when applying this antenna configuration for imaging the heterogeneously dense breast are shown in Fig. 7. The most important thing to notice in these images is how the system is less capable of reconstructing the top part of the imaging domain and both the tumor and the contuor of the breast are less well-defined than when the horizontal antenna configuration is applied. Meanwhile, the bottom part of the breast as 


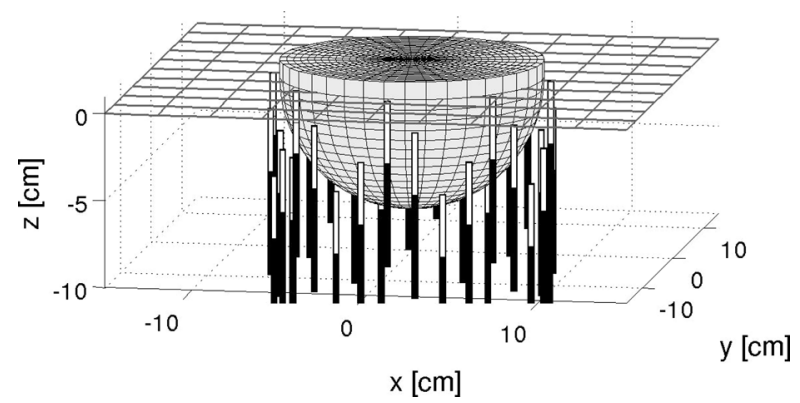

Fig. 6. Schematic of antenna system with vertically oriented antennas. The exposed dielectric of the antennas are represented by the white lines while the outer conductor of the coaxial lines are represented by the black lines.

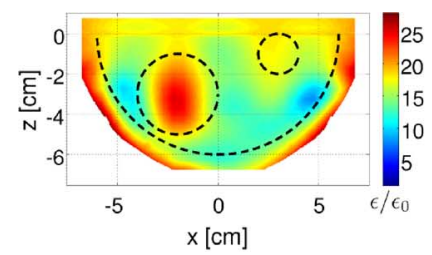

(a) Perm., $y=0 \mathrm{~cm}$

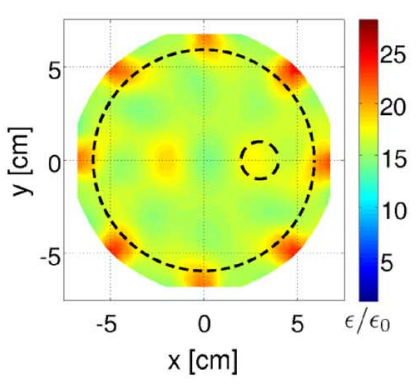

(c) Perm., $z=-1 \mathrm{~cm}$

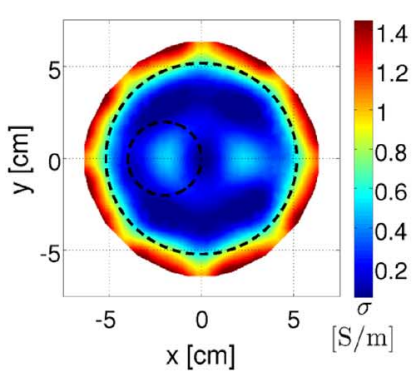

(e) Perm., $z=-3 \mathrm{~cm}$

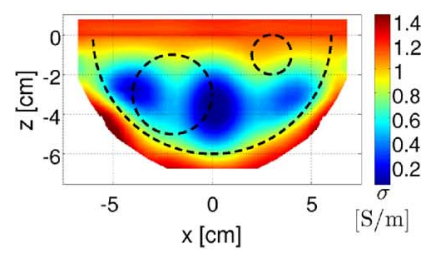

(b) Cond., $y=0 \mathrm{~cm}$

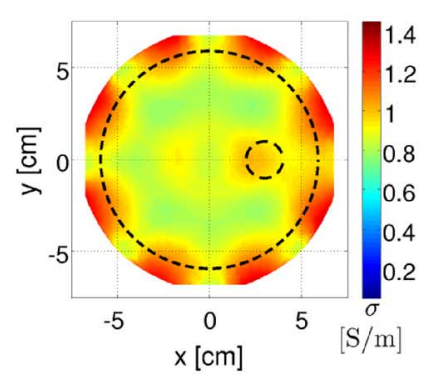

(d) Cond., $z=-1 \mathrm{~cm}$

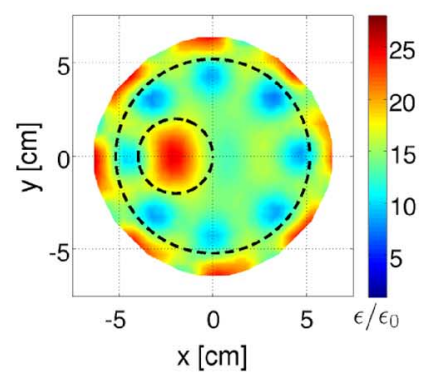

(f) Cond., $z=-3 \mathrm{~cm}$
Fig. 7. Images obtained using the vertically oriented antennas and the logphase formulation. The system is not capable of imaging the top part of the imaging domain, thereby missing the tumor. The dashed lines indicate the positions of the breast, the tumor, and the fibroglandular inclusion.

well as the fibroglandular inclusion are reconstructed with permittivity values close to the actual values.

To understand why the system is not capable of reconstructing the upper part of the imaging domain when using the vertically oriented antennas it is useful to observe the spatial distribution of the elements in the Jacobian matrix. This is a measure of how sensitive the measured signals are to changes in the constitutive parameters of the imaging domain. The spatial distribution of the Jacobians for two antennas in the top rows of the vertical and horizontal setups are shown in Fig. 8. In both cases, the transmitting antenna is located to the right while the receiving antenna

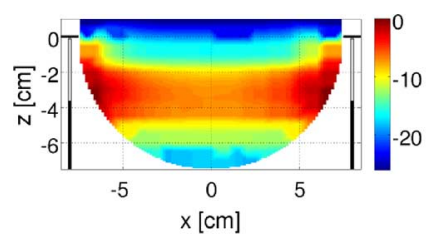

(a) Vertical

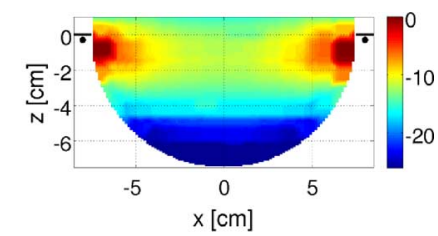

(b) Horizontal
Fig. 8. Spatial distribution of the normalized amplitude of the elements of the Jacobians between antennas on opposite sides of the imaging system for the two different antenna configurations in the plane $y=0 \mathrm{~cm}$. The antennas and the metallic lid of the imaging system are indicated in the figures. All values are in dB.

is to the left, on the opposite side of the imaging system. For the vertically oriented antennas, the normalized amplitude of the elements of the Jacobian matrix is seen to be at a maximum in an approximately $3 \mathrm{~cm}$ tall region across the imaging domain ranging from $z \approx-2 \mathrm{~cm}$ to $z \approx-5 \mathrm{~cm}$. Below and, more importantly, above this region, the amplitude of the elements of the Jacobian is seen to be significantly lower than inside the region. This implies that the system is much more sensitive to changes in the constitutive parameters within the $3 \mathrm{~cm}$ tall region than to changes outside. And while the part of the imaging domain which is located below the region is covered by other combinations of transmit- and receive antennas, no other antenna combination covers the upper part of the imaging domain better than the one showed here. Hence, any scattering objects in the lower part of the imaging domain will have a greater impact on the measured signals and thus be given more weight in the reconstruction algorithm than scatterers in the top part. This explains why the algorithm is not capable of accurately reconstructing the upper part of the imaging domain containing the tumor.

For the horizontally oriented antennas, on the other hand, the Jacobian for the two antennas is seen to have good coverage in the top part of the imaging domain while the amplitude of the elements of the Jacobian decreases towards the bottom part of the imaging domain. Similar to the setup using vertically oriented antennas, the bottom of the imaging domain is covered by other antenna combinations. This implies that the sensitivity of the system is more evenly distributed throughout the imaging domain and as a result, the algorithm is capable of reconstructing both the upper and the lower part of the imaging domain.

In both configurations, the maximum amplitude of the elements of the Jacobian are seen to occur close to the end of the outer conductor of the coaxial antennas, i.e., at the center of the antennas. This implies that the added noise in the simulated signals is likely to cause artifacts at these positions, as can be observed in all of the reconstructed images in Figs. 4, 5 and 7.

As a final observation regarding the antenna configuration, the effects of the added noise should be noted. The noise is seen to have less impact, i.e., resulting in fewer artifacts, on the images obtained using the vertically oriented antennas than on the images obtained using the horizontally oriented antennas. This is due to the fact that the coupling between the antennas is better when all of the antennas are $\hat{z}$ polarized than when they $\hat{\phi}$-directed as in the horizontal setup. Hence, the simulated noise floor $110 \mathrm{~dB}$ below the transmitted power has less effect on the reconstruction using the vertically oriented antenna setup. 
The simulation setup presented here is chosen to illustrate the performance of the TUD imaging system in what is considered to be a worst-case scenario. First, because of the large difference in constitutive parameters between the coupling liquid and the breast, the scattered field created by this contrast dominates the measurements. In clinical trials a 90:10 glycerin-water coupling liquid with a relative permittivity of approximately 14 and a conductivity of approximately $0.9 \mathrm{~S} / \mathrm{m}$ will be used as the standard coupling liquid. The parameters of this liquid are close to the average of the values reported in [11] for the breast tissue and will thus allow for more energy to be coupled to the interior of the breast. Another approach is to image each patient with two or three different liquids to better cover the large range of permittivity and conductivity values reported. This will, however, increase the examination time.

Second, the tumor is positioned close to the top of the imaging domain. This implies that only the measurements involving the top row of antennas have a notable contribution from the tumor while in the vast majority of the measurements, the tumor response is insignificant. The effects of this are further enhanced by the presence of the fibroglandular inclusion in the center of the breast. In most of the measurements, the response from this inclusion is far greater than the tumor response. Improvements may also be obtained by using a priori knowledge about the chest wall. As seen in the images obtained with the horizontally oriented antennas in Fig. 4, the chest wall is not reconstructed properly and this gives rise to additional artifacts in the images. If in clinical trials it is found that the chest wall is similar from patient to patient, either a fixed-valued chest wall can be applied in the algorithm, or the region above the lid may be assigned initial constitutive parameters in the reconstruction algorithm which are close to those of the chest wall.

Finally, the reconstruction is performed at the rather low frequency $1.3 \mathrm{GHz}$, because the values in [11] were given at this frequency. By increasing the frequency, a better resolution may be obtained and smaller objects imaged.

\section{CONCLUSION}

The TUD microwave imaging system for breast-cancer screening was presented. The imaging system consists of 32 antennas in a cylindrical setup and is designed to operate at a single frequency in the range from $300 \mathrm{MHz}$ to $3 \mathrm{GHz}$. The large frequency range allows for choosing the optimum frequency at which to do the image reconstruction.

The reconstruction algorithm is based on an iterative Newton scheme in which the measured data is represented using the $\log$-phase formulation. This has been shown to improve the performance of the imaging algorithm with a minimum of additional computation time. The updates in the Newton algorithm are calculated using a CGLS algorithm and the number of CGLS iterations is increased as the algorithm approaches convergence, corresponding to a reduction in the regularization of the solution of the linear update problems. The Newton algorithm requires the calculation of the scattered field in each iteration. To this end, a method of moment (MoM) solver has been implemented in which the time consuming operation of filling the coefficient matrix needs only to be done once after which the matrix may be stored and reused in the following Newton iterations. Furthermore, the MoM code has been augmented using the adaptive integral method which drastically reduces the memory consumption.

To illustrate the performance of the system, a model of a heterogeneously dense breast with a large fibroglandular inclusion and a smaller tumor was used. In the images of this simulated data set reconstructed using the log-phase formulation, the breast, tumor, and fibroglandular inclusions were all visible. Although the tumor in this reconstruction was not reconstructed with the actual values of permittivity and conductivity, the fact that the tumor was reconstructed with the correct size and only a small error in the position, along with a raised conductivity indicating a tumor, shows that the imaging system is capable of detecting tumors in less-than-ideal imaging conditions.

When using the complex formulation for the reconstruction, the algorithm failed to reconstruct the distribution of the constitutive parameters because of the inability of the complex formulation to handle phase changes greater than $\pm \pi$.

In addition to a comparison of the log-phase and complex formulations of the imaging problem, the effects of changing the antenna setup were investigated. Here, it was found that it is of great importance to organize the antennas of the imaging system in a suitable way to make sure the entire imaging domain is covered by the antennas.

In this paper, the system has been simulated at the frequency $1.3 \mathrm{GHz}$ since average values for the normal and fibroglandular breast tissue was available for this frequency. The system is, however, capable of working at frequencies up to $3 \mathrm{GHz}$, thereby providing better resolution. At the time of writing, research is being focused on completing the microwave circuitry for the imaging system. When phantom measurements are done, more accurate estimates of the resolution of the system will be available. Furthermore, it will be investigated whether using multiple frequency measurements improves the performance of the system.

\section{ACKNOWLEDGMENT}

The authors thank Dr. P. M. Meaney of Dartmouth College for many fruitful discussions.

\section{REFERENCES}

[1] American Cancer Society, Inc., Cancer Facts \& Figures 2007 American Cancer Society, 2007.

[2] Sundhedsstyrelsen, Cancerregisteret 2001 (Foreløbige tal for 2002-2003). Copenhagen, Denmark: Sundhedsstyrelsen - Danish National Board of Health, 2005, Nye tal fra Sundhedsstyrelsen.

[3] Sundhedsstyrelsen, Kraftstatistik Baseret på Landspatientregisteret 2000-2004. Copenhagen, Denmark: Sundhedsstyrelsen - Danish National Board of Health, 2006, Nye tal fra Sundhedsstyrelsen.

[4] D. M. Parkin, "Global cancer statistics in the year 2000," Lancet Oncology, vol. 2, no. 9, pp. 533-543, 2001.

[5] J. S. Michaelson, M. Silverstein, J. Wyatt, G. Weber, R. Moore, E. Halpern, D. B. Kopans, and K. Hughes, "Predicting the survival of patients with breast carcinoma using tumor size," Cancer, vol. 95 , no. 4 , p. 713, 2002.

[6] A. I. Mushlin, R. W. Kouides, and D. E. Shapiro, "Estimating the accuracy of screening mammography: A meta-analysis," Amer. J. Prevent. Med., vol. 14, no. 2, pp. 143-153, 1998.

[7] S. J. Nass, C. Henderson, and J. C. Lashof, Eds., Mammography and Beyond: Developing Techniques for the Early Detection of Breast Cancer Washington, DC, National Academy Press, 2000. 
[8] T. S. England and N. A. Sharples, "Dielectric properties of the human body in the microwave region of the spectrum," Nature, vol. 163, pp. 487-488, 1949.

[9] T. S. England, "Dielectric properties of the human body for wavelength in the 1-10 cm range," Nature, vol. 1466, pp. 480-481, 1950.

[10] W. T. Joines, Y. Zhang, C. Li, and R. L. Jirtle, "The measured electrical properties of normal and malignant human tissues from 50 to 900 MHz," Med. Phys., vol. 21, no. 4, pp. 547-50, 1994.

[11] P. Meaney, M. Fanning, T. Raynolds, C. Fox, Q. Fang, C. Kogel, S. Poplack, and K. Paulsen, "Initial clinical experience with microwave breast imaging in women with normal mammography," Academic Radiol., vol. 14, no. 2, pp. 207-218, 2007.

[12] S. P. Poplack, T. D. Tosteson, W. A. Wells, B. W. Pogue, P. M. Meaney, A. Hartov, C. A. Kogel, S. K. Soho, J. J. Gibson, and K. D. Paulsen, "Electromagnetic breast imaging: Results of a pilot study in women with abnormal mammograms," Radiol. - Radiolog. Society of North Amer., vol. 243, no. 2, pp. 350-359, 2007.

[13] M. Lazebnik, L. McCartney, D. Popovic, C. B. Watkins, M. J. Lindstrom, J. Harter, S. Sewall, A. Magliocco, J. H. Booske, M. Okoniewski, and S. C. Hagness, "A large-scale study of the ultrawideband microwave dielectric properties of normal breast tissue obtained from reduction surgeries," Phys. Med. Biol., vol. 52, no. 10, 2007.

[14] M. Lazebnik, D. Popovic, L. McCartney, C. B. Watkins, M. J. Lindstrom, J. Harter, S. Sewall, T. Ogilvie, A. Magliocco, T. M. Breslin, W. Temple, D. Mew, J. H. Booske, M. Okoniewski, and S. C. Hagness, "A large-scale study of the ultrawideband microwave dielectric properties of normal, benign and malignant breast tissues obtained from cancer surgeries," Phys. Med. Biol., vol. 52, no. 20, pp. 6093-6115, 2007.

[15] R. Nilavalan, J. Leendertz, I. Craddock, A. Preece, and R. Benjamin, "Numerical analysis of microwave detection of breast tumours using synthetic focussing techniques," in Proc. IEEE Antennas Propag. Society Int. Symp., 2004, vol. 3, pp. 2440-2443.

[16] X. Li and S. Hagness, "A confocal microwave imaging algorithm for breast cancer detection," IEEE Microw. Wireless Compon. Lett., vol. 11, pp. 130-132, 2001.

[17] E. Fear and M. Stuchly, "Microwave system for breast tumor detection," IEEE Microw. Guided Wave Lett., vol. 9, pp. 470-472, 1999.

[18] Y. Xie, B. Guo, L. Xu, J. Li, and P. Stoica, "Multistatic adaptive microwave imaging for early breast cancer detection," IEEE Trans. Biomed. Eng., vol. 53, pp. 1647-1657, 2006.

[19] M. Klemm, I. J. Craddock, J. Leendertz, A. Preece, and R. Benjamin, "Breast cancer detection using symmetrical antenna array," presented at the Proc. Eur. Conf. on Antennas Propag. EuCAP, 2007.

[20] E. Fear, X. Li, S. Hagness, and M. Stuchly, "Confocal microwave imaging for breast cancer detection: Localization of tumors in three dimensions," IEEE Trans. Biomed. Eng., vol. 49, pp. 812-822, 2002.

[21] E. Fear, J. Sill, and M. Stuchly, "Experimental feasibility study of confocal microwave imaging for breast tumor detection," IEEE Trans. Microw. Theory Tech., vol. 51, pp. 887-892, 2003.

[22] P. Kosmas and C. Rappaport, "Use of the FDTD method for time reversal: Application to microwave breast cancer detection," in Proc. SPIE - The Int. Society for Opt. Engineer., 2004, vol. 5299, pp. 1-9.

[23] C. Rappaport, "Cardiac tissue ablation with catheter-based microwave heating," Int. J. Hyperthermia, vol. 20, no. 7, p. 769, 2004.

[24] Q. H. Liu, C. Yu, J. Stang, M. Yuan, E. Bresslour, R. T. George, G. Ybarra, and W. T. Joines, "Experimental and numerical investigations of a high-resolution 3D microwave imaging system for breast cancer detection," in Proc. IEEE Antennas Propag. Society Int. Symp., 2007, pp. 2192-2192.

[25] P. Hashemzadeh, A. Fhager, and M. Persson, "Experimental investigation of an optimization approach to microwave tomography," Electromagn. Biol. Med., vol. 25, no. 1, pp. 1-12, 2006.

[26] A. Fhager, P. Hashemzadeh, and M. Persson, "Reconstruction quality and spectral content of an electromagnetic time-domain inversion algorithm," IEEE Trans. Biomed. Eng., vol. 53, pp. 1594-1604, 2006.

[27] P. Meaney, K. Paulsen, J. Chang, M. Fanning, and A. Hartov, "Nonactive antenna compensation for fixed-array microwave imaging. II. imaging results," IEEE Trans. Medical Imaging, vol. 18, pp. 508-518, 1999.

[28] P. Meaney, K. Paulsen, A. Hartov, and R. Crane, "Microwave imaging for tissue assessment: Initial evaluation in multitarget tissue-equivalent phantoms," IEEE Trans. Biomed. Eng., vol. 43, pp. 878-890, 1996.

[29] S. Semenov, R. Svenson, A. Bulyshev, A. Souvorov, A. Nazarov, Y. Sizov, A. Pavlovsky, V. Borisov, B. Voinov, G. Simonova, A. Starostin, V. Posukh, G. Tatsis, and V. Baranov, "Three-dimensional microwave tomography: Experimental prototype of the system and vector born reconstruction method," IEEE Trans. Biomed. Eng., vol. 46, pp. 937-946, 1999.
[30] P. Meaney, M. Fanning, D. Li, S. Poplack, and K. Paulsen, "A clinical prototype for active microwave imaging of the breast," IEEE Trans. Microw. Theory Tech., vol. 48, pp. 1841-1853, 2000.

[31] S. Semenov, R. Svenson, A. Bulyshev, A. Souvorov, A. Nazarov, Y. Sizov, V. Posukh, A. Pavlovsky, P. Repin, A. Starostin, B. Voinov, M. Taran, G. Tatsis, and V. Baranov, "Three-dimensional microwave tomography: Initial experimental imaging of animals," IEEE Trans. Biomed. Eng., vol. 49, pp. 55-63, 2002.

[32] S. Y. Semenov, A. E. Bulyshev, V. G. Posukh, Y. E. Sizov, T. C. Williams, and A. E. Souvorov, "Microwave tomography for detection/ imaging of myocardial infarction. I. Excised canine hearts," Annals Biomed. Eng., vol. 31, no. 3, pp. 262-270, 2003.

[33] P. Meaney, K. Paulsen, A. Hartov, and R. Crane, "An active microwave imaging system for reconstruction of 2-D electrical property distributions," IEEE Trans. Biomed. Eng., vol. 42, pp. 1017-1026, 1995.

[34] P. Meaney, K. Paulsen, and J. Chang, "Near-field microwave imaging of biologically-based materials using a monopole transceiver system," IEEE Trans. Microw. Theory Tech., vol. 46, pp. 31-45, 1998.

[35] P. Meaney, K. Paulsen, J. Chang, and M. Fanning, "Initial microwave imaging experiments in ex-vivo breast tissue," in Proc. First Joint BMES/EMBS Conf., 1999, vol. 2, p. 1130.

[36] D. Li, P. Meaney, and K. Paulsen, "Conformal imaging with a non-contacting microwave antenna array," in Proc. IEEE MTT-S Int. Microw. Symp., 2001, vol. 1, pp. 563-566.

[37] D. Li, P. Meaney, T. Raynolds, S. Pendergrass, M. Fanning, and K. Paulsen, "A broadband microwave breast imaging system," in Proc. IEEE 29th Ann. Bioengineer. Conf., 2003, pp. 83-84.

[38] P. Meaney, S. Pendergrass, M. Fanning, D. Li, and K. Paulsen, "Importance of using a reduced contrast coupling medium in 2D microwave breast imaging," J. Electromagn. Waves Applicat., vol. 17, no. 2, pp. 333-355, 2003.

[39] Q. Fang, P. Meaney, S. Geimer, A. Streltsov, and K. Paulsen, "Microwave image reconstruction from 3-D fields coupled to 2-D parameter estimation," IEEE Trans. Med. Imaging, vol. 23, pp. 475-484, 2004.

[40] P. Meaney, Q. Fang, M. Fanning, S. Pendergrass, T. Raynolds, C. Fox, and K. Paulsen, "Microwave breast imaging with an under-determined reconstruction parameter mesh," in Proc. IEEE Int. Symp. on Biomed. Imaging: Macro to Nano, 2004, pp. 1369-1372.

[41] P. Meaney, K. Paulsen, S. Geimer, S. Haider, and M. Fanning, "Quantification of 3-D field effects during 2-D microwave imaging," IEEE Trans. Biomed. Eng., vol. 49, pp. 708-720, 2002.

[42] P. Meaney, K. Paulsen, B. Pogue, and M. Miga, "Microwave image reconstruction utilizing log-magnitude and unwrapped phase to improve high-contrast object recovery," IEEE Trans. Medical Imaging, vol. 20, pp. 104-116, 2001.

[43] J. Sill and E. Fear, "Tissue sensing adaptive radar for breast cancer detection: Study of immersion liquids," Electron. Lett., vol. 41, no. 3, pp. 113-115, 2005.

[44] Q. Fang, P. Meaney, and K. Paulsen, "Microwave image reconstruction of tissue property dispersion characteristics utilizing multiple-frequency information," IEEE Trans. Microw. Theory Tech., vol. 52, pp. 1866-1875, 2004.

[45] G. Bozza, C. Estatico, A. Massa, M. Pastorino, and A. Randazzo, "Short-range image-based method for the inspection of strong scatterers using microwaves," IEEE Trans. Instrum. Meas., vol. 56, pp. 1181-1188, 2007.

[46] C. T. Kelley, Solving Nonlinear Equations with Newton's Method. Philadelphia, PA: SIAM, 2003.

[47] P. M. Meaney, T. Zhou, S. A. Geimer, and K. D. Paulsen, "Implications of reduced measurement data sets on overall microwave tomographic image quality," presented at the Proc. Eur. Conf. on Antennas Propag. EuCAP, 2007.

[48] S. Zainud-Deen, W. Hassen, E. Ali, K. Awadalla, and H. Sharshar, "Breast cancer detection using a hybrid finite difference frequency domain and particle swarm optimization techniques," in Proc. National Radio Sci. Conf., 2008, pp. 1-8.

[49] P. Meaney, K. Paulsen, and T. Ryan, "Two-dimensional hybrid element image reconstruction for TM illumination," IEEE Trans. Antennas Propag., vol. 43, pp. 239-247, 1995.

[50] J. Troelsen, P. Meincke, and O. Breinbjerg, "Frequency sweep of the field scattered by an inhomogeneous structure using method of moments and asymptotic waveform evaluation," in IEEE Antennas Propag. Society Int. Symp. Digest, 2000, vol. 3, pp. 1830-1833.

[51] E. Jørgensen, J. L. Volakis, P. Meincke, and O. Breinbjerg, "Higher order hierarchical Legendre basis functions for electromagnetic modeling," IEEE Trans. Antennas Propag., vol. 52, pp. 2985-2995, Nov. 2004. 
[52] O. S. Kim and P. Meincke, "Adaptive integral method for higher order method of moments," IEEE Trans. Antennas Propag., vol. 56, pp. 2298-2305, 2008.

[53] T. Rubaek and P. Meincke, "Including antenna models in microwave imaging for breast cancer screening," in Proc. 1st Eur. Conf. on Antennas Propag., 2006, pp. 1-6.

[54] T. Rubæk, P. M. Meaney, P. Meincke, and K. D. Paulsen, "Nonlinear microwave imaging for breast-cancer screening using Gauss-Newton's method and the CGLS inversion algorithm," IEEE Trans. Antennas Propag., vol. 55, pp. 2320-2331, 2007.

[55] P. C. Hansen, Rank-Deficient and Discrete Ill-Posed Problems: Numerical Aspects of Linear Inversion. Philadelphia, PA: SIAM, 1998, Monographs on Mathematical Modeling and Computation.

[56] C. T. Kelley, Iterative Methods for Linear and Nonlinear Equations. Philadelphia, PA: SIAM, 1995, vol. 16, Frontiers in Applied Mathematics.

[57] J. M. Ortega and W. C. Rheinboldt, Iterative Solution of Nonlinear Equations in Several Variables, R. E. O'Malley, Ed. Philadelphia, PA: SIAM, 2000, Classics in Applied Mathematics.

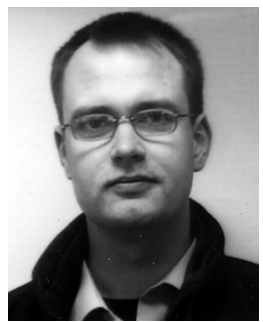

Tonny Rubæk received the M.Sc.E.E. and Ph.D. degrees from the Technical University of Denmark, Lyngby, in 2004 and 2008, respectively.

His research areas include linear and non-linear microwave-imaging algorithms and hardware.

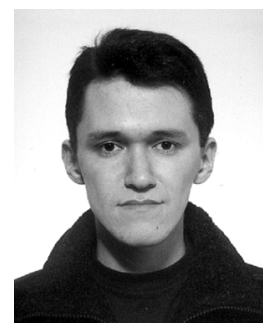

Oleksiy S. Kim received the M.S. and Ph.D. degrees from the National Technical University of Ukraine, Kiev, in 1996 and 2000, respectively, both in electrical engineering.

In 2000, he joined the Antenna and Electromagnetics Group, Technical University of Denmark (DTU), Lyngby, where he is currently an Associate Professor in the Department of Electrical Engineering. His research interests include computational electromagnetics, metamaterials, small antennas, photonic bandgap and plasmonic struc-

tures.

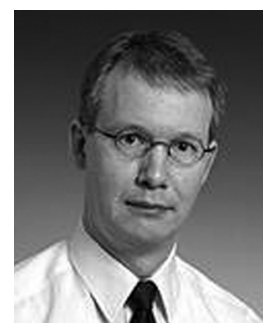

Peter Meincke was born in Roskilde, Denmark, on November 25, 1969. He received the M.Sc.E.E. and $\mathrm{Ph} . D$. degrees from the Technical University of Denmark (DTU), Lyngby, in 1993 and 1996, respectively.

In the spring and summer of 1995, he was a Visiting Research Scientist at the Electromagnetics Directorate of Rome Laboratory, Hanscom Air Force Base, MA. In 1997, he was with a Danish cellular phone company, working on theoretical aspects of radio-wave propagation. In the spring and summer of 1998, he was visiting the Center for Electromagnetics Research at Northeastern University, Boston, MA, while holding a Postdoctoral position from DTU. In 1999, he became a staff member of the Department of Electromagnetic Systems, DTU. In 2008, he joined TICRA Engineering Consultants, Copenhagen, Denmark, where he is currently employed as a Senior Engineer.

Dr. Meincke won the first prize award in the 1996 IEEE Antennas and Propagation Society Student Paper Contest in Baltimore, MD, for his paper on uniform physical theory of diffraction equivalent edge currents. He also received the 2000 R. W. P. King Paper Award for his paper entitled "Time-domain version of the physical theory of diffraction" published in the February 1999 issue of the IEEE TRANSACTIONS ON ANTENNAS AND PROPAGATION. 